\title{
Rui Pedro Paula de Matos, As ONG(D) e a crise do Estado soberano, Lisbonne Universidade Lusíada, 2001, 668 p., bibl., ISBN : 972-8397-23-3.
}

Cet ouvrage est la publication, sans aucun changement (à l'exception de bien longs remerciements de l'auteur à tous ses professeurs depuis la maîtrise) d'une épaisse thèse de mestrado (troisième cycle). L'auteur est diplômé en sciences politiques, et on doit remarquer qu'il s'agit de l'un des rares ouvrages de sciences sociales pour lesquels l'auteur affirme un tel lien disciplinaire, assez rare au Portugal. Le thème des «ONG de développement et la crise de l'État souverain » est excellent. Le livre, non, pour des raisons tant de forme que de fond.

On doit d'abord questionner la praticabilité de la publication, en livre et sans modification, de thèses lourdes de caractéristiques très «scolaires », où l'étudiant cherche à montrer qu'il a lu tout ce qu'il fallait et est capable de définir tous les concepts présents dans chaque phrase. Ainsi, dans une œuvre sur les ONG et la société civile, c'est à la page 440 que l'auteur nous dit: "Après avoir exposé quelques idées au sujet de la société civile, il convient [sic, port. : é conveniente] d'évaluer dans quelle mesure ce concept peut être opérationnel dans le Portugal contemporain ». Bonne idée en effet, quoique tardive. De même, pour un livre considéré comme achevé, il n'est pas supportable que la moitié de la totalité du texte soit en notes de bas de page. Ou c'est utile et dans le corps du texte, ou ce ne l'est pas et saute (sauf, évidemment, les références et remarques brèves).

La bibliographie est impressionnante (pp. 631-660) et internationale, c'est certain. Mais elle est uniquement une bibliographie de sources secondaires, il n'y a aucune source primaire : pas une entrevue d'un activiste d'une ONG, pas d'archives d'ONG, pas de journaux d'ONG à de rares exceptions près (par exemple, la Plataforma das ONGDs portuguesas). Il n'y a pas d'histoire critique des ONG au Portugal, pas d'analyse de leur activité concrète au Portugal et dans les Pays africains de langue officielle portugaise, que l'auteur n'a pas visités. Le livre est uniquement une réflexion fondée sur la bibliographie de ce que d'autres ont écrit sur les ONG, la société civile, l'État, etc.

Même ainsi, de nombreuses questions ne sont pas abordées: le lien entre la privatisation néolibérale et la mode des ONG; la dépendance des ONG envers les donateurs gouvernementaux et intergouvernementaux; la confusion entre le mouvement des ONG et l'associationnisme en général (on ne voit plus de différence !) ; la «représentativité » de la société civile n’est pas relativisée, alors qu'elle apparaît pourtant comme le substitut du peuple souverain; le concept de «société civile mondiale » est largement utilisé, mais sans jamais questionner sa praticabilité en Afrique et en Asie, zones groupant la majorité de la population mondiale.

L’impression générale est donc celle, pénible, d'un encyclopédisme mal maîtrisé. Cependant, l'auteur avait des opinions et des analyses qui auraient pu être exposées de manière intéressante si le nombre de pages avait été divisé par six. Le fait de décrire, préalablement à chaque thème abordé, toute la bibliographie des sciences politiques sur le sujet, transforme la lecture en un cauchemar. C'est dommage.

Novembre 2002 et mai 2005, Michel Gahen 